ISSN 0001-6002/2002/44/3/117-120

A cta M édica Costarricense, $\bigcirc 2002$

Colegio de M édicos y Cirujanos

\title{
Diagnóstico tardío de displasia evolutiva de cadera en la población infantil costarricense en el periodo 1996-2000
}

\author{
Cecilia Monge-Bonilla ${ }^{1}$, Adriana Sánchez-Ramírez¹, Catalina Morales-Alpízar ${ }^{1}$, Pablo Serrano-Aybar ${ }^{1}$, \\ Róger López-Ruiz'
}

Resumen: En este proyecto se plantea un problema de repercusión en la salud pública: el diagnóstico tardío de Ia DEC. El objetivo general del estudio es determinar los casos de DEC diagnosticados tardadamente en el periodo establecido, que fueron internados en el $\mathrm{HNN}$, con el fin de concienciar a la comunidad médica sobre la magnitud del problema, sus complicaciones potencialmente irreversibles y enfatizar la importancia de la detección temprana.

M étodos: Este es un estudio retrospectivo que analiza expedientes médicos de pacientes internados con el diagnóstico de DEC en el HNN, de 1996 a 2000. La muestra fue escogida de manera aleatoria. Los datos se organizaron en un instrumento de recolección y se analizaron de acuerdo con tasas, promedios, porcentajes y relaciones entre ellos.

Resultados: La mayor incidencia de casos de DEC se da en San José, seguida por Cartago y en tercer lugar, Heredia. La mayoría de los pacientes fueron diagnosticados en el periodo comprendido entre el primero y los cinco años. La edad promedio de la muestra fue de 3 años y 4 meses, con una desviación estándar de 4.99.

Se encontró que el $82 \%$ de los pacientes fueron del sexo femenino y el $18 \%$ del masculino. El tipo de luxación más frecuente fue la izquierda, en un $55.2 \%$ de los casos; la luxación derecha correspondió a un $24.8 \%$, y la bilateral a un 20.0\%. La relación entre izquierda y derecha fue de 2.2:1.

A I nacer, presentación fetal más frecuente fue la cefálica, en un $62.9 \%$ de los pacientes. La presentación pélvica representó, un $26.7 \%$ y en un $10.5 \%$ de los afectados, no se indicó. En cuanto al tipo de parto, un $80 \%$ fue vaginal y un $19 \%$ por cesárea, en un $7 \%$, no se indica.

El porcentaje de niños nacidos de término fue de un $67.6 \%$

Conclusiones: Dentro de la población de estudio, la mayor parte de los pacientes fueron diagnosticados después del año, cuando el defecto se hizo evidente por el inicio de la bipedestación ${ }^{11}$. De toda la población de estudio, el $98.5 \%$ fue sometida a al gún tipo de tratami ento quirúrgico. L a intervención quirúrgica, además de representar un al to costo institucional, constituye un trauma para el paciente, los resultados no siempre son óptimos y las complicaciones son diversas.

Palabras clave: Displasia evolutiva cadera, diagnóstico

Recibido: 25 de febrero del 2002

Aceptado: 21 de mayo del 2002

La Displasia Evolutiva de Cadera (DEC) es el defecto congénito más frecuente en los recién nacidos, en escala

Abreviaturas: DEC, Displasia evolutiva de cadera, HNN, Hospital Nacional de Niños, DDH , Developmental Displasia of the Hip

Servicio de Ortopedia, Hospital Nacional de Niños “Dr. Carlos Sáenz Herrera".

Correspondencia: A driana Sánchez Ramírez. A partado Postal 577 La U ruca. San J osé, Costa Rica. Correo electrónico: A drianasanchez@ hotmail.com mundial, con una incidencia de 5 por cada 1000 nacidos vivos'. En Costa Rica es de 6 por cada 1000 nacidos vivos, según el trabajo epidemiológico de la Dra. Ramírez y otros, siendo de las más altas en el mundo. Ocupa el primer lugar en frecuencia, como malformación congénita en nuestro país.

Este es el tema de mayor discusión en la literatura ortopédica pediátrica. Su diagnóstico temprano es de suma importancia para evitar complicaciones en el infante, como la artrosis coxofemoral. 
DEC es el término utilizado para describir la alteración o patología que afecta el acetábulio de la cabeza femoral o el tercio proximal del fémur, que puede o no estar separado 0 luxado9. Factores genéticos están involucrados en la etiología de esta enfermedad ${ }^{3}$ Además, existen factores de riesgo, fuertemente asociados con esta condición, que son: género femenino, primigravidez, presentación pélvica, oligohidramnios ${ }^{3}$.

El diagnóstico de la enfermedad es inicialmente clínico, y el médico de atención primaria juega el papel más importante. El diagnóstico debe realizarse durante los tres primeros meses de vida. En este periodo hay ciertos signos clínicos específicos que ayudan al médico a realizar el diagnóstico, como el signo de Ortolani, que es la entrada de la cadera dislocada dentro del acetábulo ${ }^{6}$. También se cuenta con la maniobra de Barlow, que se realiza al aducir la cadera, si la cadera; sale de su posición en el acetábulo se habla de una cadera dislocable que es un signo de Barlow positivo6. Después del lapso entre las 8 y las 12 semanas de edad, estos signos ya no son útiles. El indicador más fidedigno de una cadera dislocada en un infante de tres meses es la limitación a la abducción, debido a que disminuye la laxitud de la cápsula articular y aumenta la tensión de los músculos aductores ${ }^{10}$. Cuanto más temprano se realice el diagnóstico de DEC, más simple y efectivo será el tratamiento.

\section{Materiales y métodos}

La investigación se basa en un estudio retrospectivo que analiza expedientes médicos de pacientes internados en el Hospital Nacional de Niños "D octor Carlos Sáenz Herrera" (HNN ), entre los años 1996 y 2000. La población de estudio son todos los pacientes con internamientos desde el 1 de enero de 1996 hasta el 31 de diciembre de 2000, que tienen como diagnóstico de egreso, luxación congénita de cadera. De esta población se obtuvo una muestra randomizada:

Se revisó la lista de manera que no se repitiera el expediente médico. Dentro de esta ecuación, " $x$ " corresponde al número total de pacientes, $\mathrm{y}$ " $\mathrm{N}$ " es la cantidad de expedientes que se

\begin{tabular}{|c|c|}
\hline \multicolumn{2}{|c|}{$\begin{array}{c}\text { Cuadro } 1 \\
\text { Tasa de casos de DEC diagnosticados } \\
\text { tardiamente en el Hospital Nacional de Niños, } \\
\text { según provincia, en el periodo 1996-2000 }\end{array}$} \\
\hline Provincia & Tasa por 100000 habitantes \\
\hline San José & 3.5 \\
\hline Alajuela & 2.7 \\
\hline Cartago & 3.2 \\
\hline Heredia & 3.1 \\
\hline Guanacaste & 0.37 \\
\hline Puntarenas & 2.8 \\
\hline Limón & 0.88 \\
\hline \multicolumn{2}{|c|}{$\begin{array}{l}\text { Fuente: Instrumento de recolección de datos y Censo Poblacional de } \\
\text { Costa Rica, según provincia, del año } 2000 \text {, INEC. }\end{array}$} \\
\hline
\end{tabular}

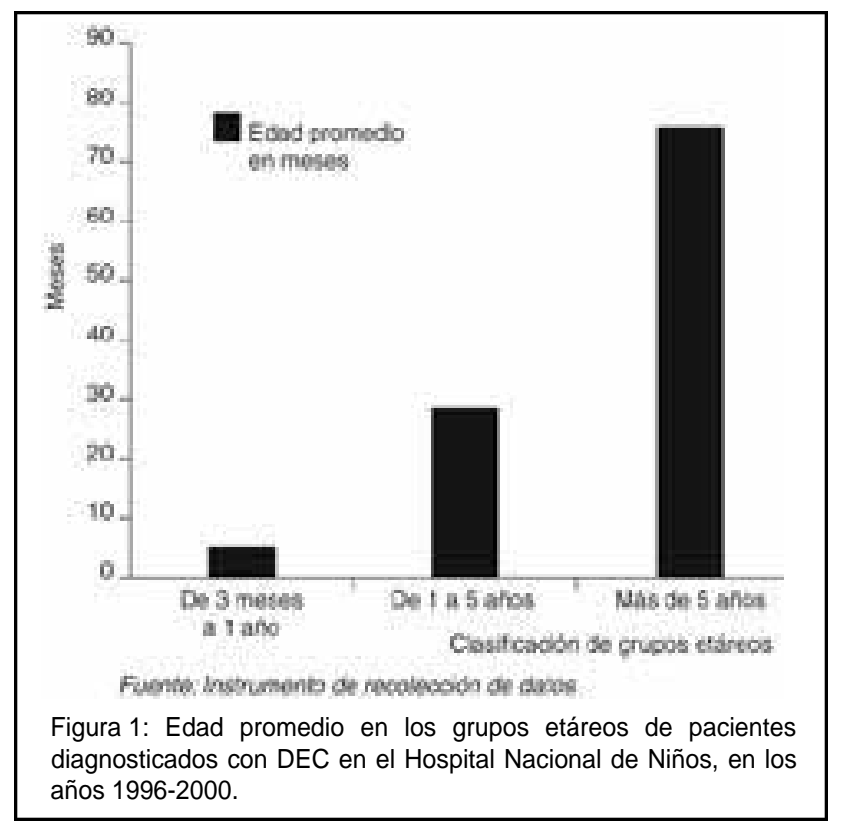

deben utilizar para obtener una muestra estadísticamente significativa; "188" es una constante. A I resul tado se le suma el $6 \%$, que corresponde a falla de información. Luego se divide el número de expedientes por el total de años que cubre el estudio. La escogencia de los expedientes, por año, se realiza en forma aleatoria.

Se definió "caso" como aquellos niños con los siguientes criterios de inclusión: internamientos desde el 1 de enero de 1996 hasta el 31 de diciembre de 2000, diagnóstico de egreso, luxación congénita de cadera, con una edad de 3 meses o mayor.

Para el propósito de este estudio se utilizó un instrumento para recolectar datos, con información obtenida de los expedientes médicos.

El protocolo de investigación fue revisado y aprobado por el Comité ético-científico del HNN.

\section{Resultados}

L os resultados presentados en la figura 1, correspondientes a los tres grupos etáreos de diagnóstico, se describen utilizando el promedio de la edad y su respectiva desviación estándar, como medidas de tendencia central.

En el cuadro 1 se muestra la frecuencia de DEC diagnosticada tardíamente, representada en tasas según provincia de procedencia, que recibió tratamiento en el periodo comprendido entre 1996 y 2000, en el HNN. Cabe destacar que el mayor número de casos procedió de San J osé, seguido por Cartago y en tercer lugar Heredia.

En la figura 1 se muestra el porcentaje de pacientes diagnosticados tardíamente distribuidos en tres periodos. La mayor parte de los pacientes fueron diagnosticados entre el primero y los cinco años. 


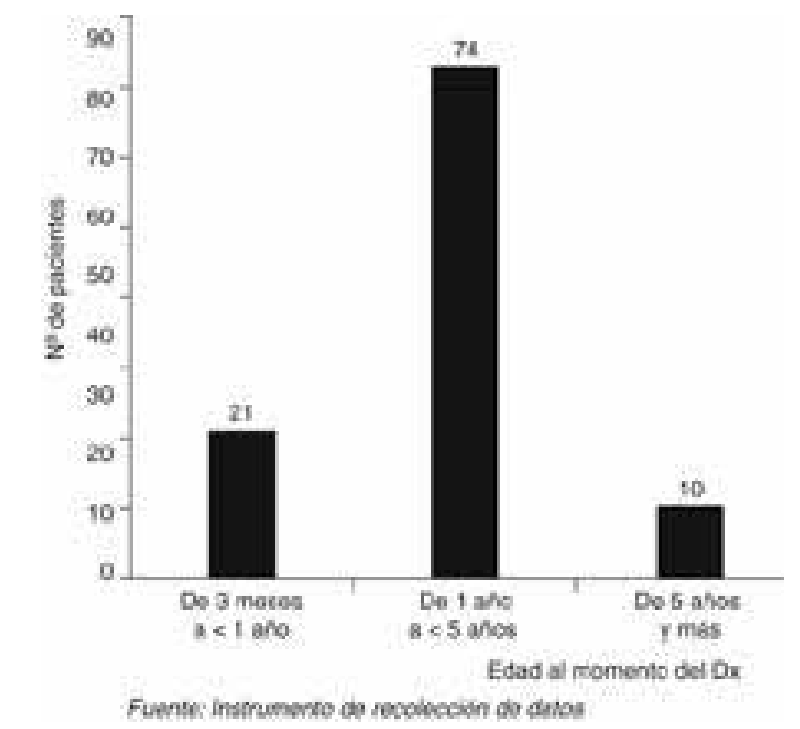

Figura 2: Diagnóstico de displasia evolutiva de cadera, según edad, en el Hospital Nacional de Niños, en los años 1996-2000.

La figura 2 presenta la edad promedio de diagnóstico en los grupos etáreos establecidos con su desviación estándar. La edad promedio de la muestra fue de 3 años y 4 meses, con una desviación estándar de 4.99 .

En el cuadro 2 se presenta la relación entre el tipo de cadera afectada, izquierda, derecha o bilateral, y género en números absolutos. Se encontró que un $82 \%$ de los afectados fueron del sexo femenino y un $18 \%$, del masculino.

L a cadera afectada con más frecuencia fue la izquierda, en un $55.2 \%$; la luxación derecha correspondió a un $24.8 \%$. Se dio afección bilateral en un $20.0 \%$ de los pacientes. La relación entre luxación izquierda y derecha fue de 2.2:1.

A I nacer, la presentación más frecuente fue la cefálica, en un $62.9 \%$ de los pacientes. La presentación pélvica representó un $26.7 \%$ y en un $10.5 \%$ de los afectados, no se indicó. En cuanto al tipo de parto, un $80 \%$ fueron por la vía vaginal y un $19 \%$, por cesárea; en un $7 \%$ no se indica.

El porcentaje de niños nacidos de término fue de un $67.6 \%$.

\section{Discusión}

En este trabajo se observó que la zona del país de la cual se refirió la mayor cantidad de casos de DEC tardía fue San J osé, seguida de Cartago y en tercer lugar, Heredia. tales datos no concuerdan con los obtenidos previamente en nuestro país, pues esos citan a Cartago como la provincia con mayor frecuencia de esta patología ${ }^{8}$. Se desconoce la razón, pero la causa genética figura entre las etiologías por investigar 1,2 . Sin embargo, los datos aportados por el INCIENSA en los últimos años y el actual estudio, demuestran que la tendencia ha cambiado, de manera que la prevalencia ha disminuido en Cartago y ha aumentado en otras provincias 4.5 .

\begin{tabular}{|lllll|}
\hline \multicolumn{5}{|c|}{$\begin{array}{c}\text { Cuadro } 2 \\
\text { Relación entre tipo de cadera afectada y sexo, en los } \\
\text { pacientes diagnosticados con DEC en el Hospital } \\
\text { Nacional de Niños, en los años 1996-2000. }\end{array}$} \\
\hline \multicolumn{5}{c}{ Cadera afectada } \\
\hline Sexo & Izquierda & Derecha & Bilateral & Total \\
Masculino & 11 & 4 & 4 & 19 \\
Femenino & 47 & 22 & 17 & 86 \\
Total & 58 & 26 & 21 & 105 \\
\hline
\end{tabular}

La frecuencia de DEC es mayor en el sexo femenino que en el masculino, con una relación de 4:1 1,910,11. En este estudio la relación concuerda con lo citado en la bibliografía. La mayor incidencia de esta patología en el sexo femenino es explicada por $\mathrm{Scott}{ }^{10}$, quien postula que las mujeres son más susceptibles que los hombres a la acción de la hormona materna relaxina, la cual puede contribuir a la laxitud ligamentosa. La relaxina, al igual que los estrógenos, bloquea la maduración de la tropocolágena recién sintetizada, lo cual afecta los enlaces cruzados y produce una hiperlaxitud ligamentosa ${ }^{11}$. A pesar de estos hallazgos, la causa exacta de la hiperlaxitud de los ligamentos es aún desconocida.

Se describe que la DEC izquierda se presenta en un $60 \%$ de los pacientes, la derecha en un $20 \%$ y un $20 \%$ es bilateral ${ }^{7}$. La relación entre afección izquierda y derecha es de 3:1 2,10. LoS resultados de esta revisión concuerdan con la bibliografía anteriormente citada. La mayor frecuencia de afectación izquierda se debe a la relación entre la posición de la cadera izquierda, del feto con el promontorio sacro de la madre, en la posición fetal occipito anterior izquierda de la mayoría con presentación cefálica ${ }^{10,11}$. De todos los nacimientos solo un $3 \%$ corresponde a presentación pélvica, un $96 \%$ son cefálicos ${ }^{5}$. La presentación pélvica in útero constituye un factor de riesgo para el desarrollo de DEC, ya que se ha demostrado en varios estudios que, de los niños afectados con esta patología, hasta un $23 \%$ tienen presentación pélvica al nacer ${ }^{11}$. Esta presentación es el factor crítico y no el recorrido por el canal de parto, pues que se ha comprobado que los niños en posición de nalgas extraídos por cesárea, tienen la misma predisposición notable a la luxación ${ }^{11}$. De las diversas presentaciones de nalgas, la que presenta mayor riesgo es la completa, con flexión de las caderas e hiperextensión de las rodillas ${ }^{2,3,10,11}$. Los datos aportados por esta revisión muestran una frecuencia similar de presentación de nalgas en pacientes con DEC, a la citada por Tachdjian ${ }^{11}$.

Existen tres periodos en los que la cadera puede estar en peligro de luxación: la semana 12, la semana 18 y las cuatro semanas finales de gestación. En este último lapso, la articulación coxofemoral y todos sus músculos están totalmente desarrollados. L os factores que producen luxación en esta fecha son fuerzas mecánicas anormales, por posición 
intrauterina defectuosa del feto, como presentación pélvica u oligohidramnios ${ }^{1,2}$. Según Tachdjian, el tipo de DEC más frecuente en el neonato, que se produce en las últimas cuatro semanas de gestación. De acuerdo con esto, se plantea en la presente investigación que el ser de término constituye un factor de riesgo para desarrollar DEC, por lo que la edad gestacional al nacer se incluyó en el instrumento de recolección de datos. La mayoría de los pacientes estudiados fueron de término; sin embargo, esta mayoría no superó el porcentaje de nacimientos de término en la población general $(78 \%)^{5}$.

Dentro de la población de estudio, la mayor parte de los pacientes fueron diagnosticados después del año, cuando el defecto se hizo evidente por el inicio de la bipedestación ${ }^{11}$. De toda la población de estudio, el $98.5 \%$ tuvo que ser sometido a algún tipo de tratamiento quirúrgico. La intervención quirúrgica además, de representar un al to costo para la institución, constituye un trauma para el paciente; con frecuencia los resultados no son óptimos y las complicaciones son diversas.

\section{Abstract}

Late diagnosis of DDH is an important public health issue, the purpose of this project is to make the medical community aware of the problem and to emphasize on early detection. The aim is to analyze the cases of DDH with delayed diagnosis, seem at the National Children's Hospital in San J osé, Costa Rica.

This is a retrospective study that analyzes medical charts of patients with DDH whose diagnosis were reached late and that were hospitalized at the National Children's Hospital between the years of 1996 and 2000. The sample was randomized. The information was organized in a data base designed for this purpose. The information was presented and analyzed by percentages, mean, standard deviation and the relationships between them.

The great majority of cases of DDH were found in San José, followed by Cartago and in third place Heredia. M ost of the patients were diagnosed between their first and fifth year of age. The mean age of diagnosis was 3 years and 4 months with a standard deviation of 4.99. From the total population, $82 \%$ of patients were female and $18 \%$ male. The most frequent type of dislocation site was left in $52 \%$ of the cases, right was found in $24.8 \%$ and bilateral in $20 \%$. The ratio between left and right dislocation was 2.2:1.
The most frequent presentation at birth was cephalic in $62.9 \%$ of the patients, $26.7 \%$ were pelvic and it was not indicated in $10.5 \%$ of cases.

Concerning the type of birth, $80 \%$ were vaginal and $19 \%$ by cesarean section, $7 \%$ of the charts did not have this information.

\section{The percentage of children born at term was $67.6 \%$}

The great majority of patients in this study were diagnosed after one year of age, when the defect became evident while walking. Of the total population, $98.5 \%$ had to be submitted to some kind of surgical treatment. Surgical intervention represents high costs for the institution, traumatic to the patient and the results are frequently not optimal with many possible complications.

\section{Referencias}

1. B ehrman M. K liegman: Developmental dysplasia of the hip. Essentials of Pediatrics W.B. Saunders, 1994; 713-717.

2. Dysplasia of the Hip. Early Detection of Developmental Dysplasia of de Hip. Pediatrics, 2000; 105: 896-905.

3. French $L$ and Dietz F. Screening for Developmental Dysplasia of the Hip. A merican A cademy of family physicians, 1999. 1:7. Disponible en http://www.aafp.org/afp/990700ap/177.html

4. Instituto costarricense de investigación y enseñanza en nutrición y salud. Malformaciones Congénitas en Costa Rica, 1999. San José, Costa Rica. 2000.

5. Instituto costarricense de investigación y enseñanza en nutrición y salud. Prerregistro de M alformaciones Congénitas en Costa Rica, 2000. San J osé, Costa Rica. 2001.

6. Lehmann $H$, Hinton $R$, M orelo P, Santoni I. Developmental Dysplasia of the Hip Practice Guideline: Technical Report. Pediatrics 2000; 105: e57.

7. Lewis K, Jones D, Powel N. Ultrasound and N eonatal Hip Screening: The Five-Y ear Results of a Prospective Study in High Risk Babies. J Pediatr Orthop 1999; 19:760-762.

8. Ramírez L. Luxación congénita de cadera de diagnóstico tardío, distribución regional en Costa Rica, 1986 a 1990. Tesis de grado. Universidad de Costa Rica. San J osé, Costa Rica. 1992.

9. Salter A. Luxación y subluxación de la cadera. En: Lange, M. Trastornos y lesiones del sistema musculoesquelético. Barcelona: Editorial Jims, 1969: 107-115.

10. Scott, S. AAP Develops Guidelines for Early Detection of Dislocated Hips. Practice Guidelines, 2001; 1:3. Disponible en http://www.aatip.org/atip/20010201/practice.html.

11. Tachdjian, M: Ortopedia Pediátrica. Buenos Aires: Editorial Interamericana, 1994; 322:564. 